\title{
Discrete-Time Complex Bandpass Filters for Three-Phase Converter Systems
}

\section{Guest, Emerson; Mijatovic, Nenad}

Published in:

IEEE Transactions on Industrial Electronics

Link to article, DOI:

10.1109/TIE.2018.2860554

Publication date:

2018

Document Version

Peer reviewed version

Link back to DTU Orbit

Citation (APA):

Guest, E., \& Mijatovic, N. (2018). Discrete-Time Complex Bandpass Filters for Three-Phase Converter Systems. IEEE Transactions on Industrial Electronics, 66(6), 4650 - 4660. https://doi.org/10.1109/TIE.2018.2860554

\section{General rights}

Copyright and moral rights for the publications made accessible in the public portal are retained by the authors and/or other copyright owners and it is a condition of accessing publications that users recognise and abide by the legal requirements associated with these rights.

- Users may download and print one copy of any publication from the public portal for the purpose of private study or research.

- You may not further distribute the material or use it for any profit-making activity or commercial gain

- You may freely distribute the URL identifying the publication in the public portal

If you believe that this document breaches copyright please contact us providing details, and we will remove access to the work immediately and investigate your claim 


\title{
Discrete-Time Complex Bandpass Filters for Three-Phase Converter Systems
}

\author{
Emerson Guest, and Nenad Mijatovic, IEEE Senior Member
}

\begin{abstract}
A first-order complex bandpass filter (CBF) derived in the discrete frequency domain is proposed as a building block for the complex signal processing unit in three-phase converter systems. The first-order discrete CBF can be directly implemented in a digital system, is stable at all center frequencies and has a low computational burden. The first-order CBF is extended to a $p$ thorder discrete CBF. A normalized frequency-locked loop $(F L L)$ is then developed that allows the frequency adaption to satisfy a desired settling time regardless of the input signal magnitude or discrete CBF form. The $p$ th-order discrete CBF-FLL was tested on an FPGA in the form of two industrial applications: voltage synchronization under grid fault and extraction of an unknown frequency component. The second-order discrete CBF-FLL offered significantly improved stopband attenuation and frequency estimation relative to the first-order discrete CBF-FLL, for a small increase in computational burden.
\end{abstract}

Index Terms-Frequency estimation, bandpass filters, digital filters.

\section{INTRODUCTION}

$\mathbf{O}$ $\mathrm{NE}$ of the recent trends in grid-connected three-phase converter systems has been to embed signal processing in the stationary-frame using bandpass filters and frequencyadaptive loops [1]-[3]. This approach is motivated by the improved converter performance during phase jump and unbalanced faults compared to systems based on synchronousframe phase-locked loops (SRF-PLLs) [4]. The most prolific stationary-frame filter schemes found in the literature are based on real-coefficient (hereafter simply referred to as real), continuous, second-order filters. Examples include the secondorder generalized integrator (SOGI) [1],[2], the adaptive notch filters (ANF) [5],[6] and the extended PLL (EPLL) [7],[8].

Stationary-frame complex filters have also emerged as a promising alternative to real filters in three-phase converter systems. Complex filters have cross-coupling between the inphase and quadrature signal paths, resulting in asymmetrical frequency responses around the zero frequency [9]. When expressed in complex single-input single-output (SISO) form, complex filters necessarily have complex coefficients [10]. Voltage synchronization schemes have been proposed based

Manuscript received February 6, 2018; revised April 2 and June 8 , 2018; accepted June 29, 2018. This work was supported in part by the Innovations Fund, Denmark.

Emerson Guest and Nenad Mijatovic are with the Center for Electric Power and Energy, Department of Electrical Engineering, Technical University of Denmark, Lyngby 2800, Denmark (e-mail: edagu@elektro.dtu.dk; nm@elektro.dtu.dk). on the first-order continuous complex bandpass filter (CBF) [11],[12]. CBFs allow a specific frequency and phase sequence to be targeted, avoiding an explicit sequence domain extractor otherwise required in SOGI, ANF and EPLL-based schemes. Furthermore, a first-order CBF requires half the number of real states compared to a real bandpass filter such as the twochannel SOGI [13]. Complex filters have also been used to provide closed-loop complex bandpass response for regulating complex current signals [14] and in impedance-based active filtering in the form of a sliding discrete Fourier transform (DFT) [15],[16].

Implementing a continuous filter in a digital signal processor, such as in [1]-[8],[11],[12],[17], inevitably requires high-order approximations of the continuous integrators. Loworder approximations have exhibited poor performance in resonant filters when applied to harmonic filtering [18]. Jorge et. al. [13], however, showed that a filter designed in the discrete frequency domain offered the same performance as the continuous filters in [2],[11] but with reduced computation time. In fact, the discrete filter proposed in [13] will be shown to be just one route for obtaining a discrete $\mathrm{CBF}$, in that case from a complex allpass filter. An alternate complex allpass filter has been used to construct a discrete complex ANF [19].

A limitation of the existing SOGI- and first-order CBFbased voltage synchronization schemes is that they rely on a harmonic decoupling network [2],[11],[12],[13] (and hence deterministic knowledge of the distortion) to achieve good magnitude, phase and frequency estimations under distorted and unbalanced conditions. In reality an effective decoupling may not be possible due to the presence of transient, noncharacteristic harmonic and interharmonic components generated by a variety of nonlinear sources and loads in the modern power system [20]. Furthermore, applications requiring cascaded filter to detect unknown frequency components [21] are incompatible with a decoupling network. The best approach is to design filter schemes that guarantee correct operation under generic grid voltage or current conditions, with special consideration to abnormal conditions (such as during grid faults) [22]. Consequently, higher-order continuous filters have been proposed as pre-filters to grid-synchronization schemes [17],[23]. These filters achieve greater stopband attenuation than their low-order counterparts for a given response time.

In this paper a $p$ th-order discrete $\mathrm{CBF}$ is proposed, unifying the specificity of the CBF in sequence component extraction with the improved stopband attenuation offered by higherorder filters, whilst at the same time keeping the computational burden low. In Section II the continuous and discrete CBFs 
are reconciled through an analysis of their zero and pole placements, highlighting which discrete forms are preferable. A normalized frequency-locked loop (CBF-FLL) is developed for the $p$ th-order discrete CBF in Section III, as required for frequency-adaptive complex signal processing in three-phase converter systems under generic grid voltage conditions. Normalization renders the frequency adaption rate approximately invariant to the input signal magnitude and discrete CBF form. The expected dynamics of the normalized FLL are analyzed using a tractable cross-spectral method [19]. Section IV covers an FPGA implementation of the CBF-FLL algorithms in the form of two industrial applications: voltage synchronization during a grid fault and separation of power system signals of unknown frequency. The second-order CBF-FLL will be shown to offer significantly improved stopband attenuation and frequency estimation relative to the first-order CBF-FLL, for a small increase in computational burden.

\section{Stationary-Frame Complex Bandpass Filters}

\section{A. Rationale for Using a Complex Bandpass Filter}

The SOGI with its extensively researched bandpass and FLL properties [1]-[3],[17],[23] is used as the starting point for the present investigation. The second-order real bandpass filter constructed by the SOGI is

$$
Q_{S O G I}(s)=\frac{k \omega_{h}^{\prime} s}{s^{2}+k \omega_{h}^{\prime} s+\omega_{h}^{\prime 2}},
$$

where $k$ is a damping factor and $\omega_{h}^{\prime}$ is the natural frequency. In three-phase applications the main signal processing objective is extracting a sequence component of frequency $\omega_{h}$. This can be achieved through coupling of the direct and quadrature signals [1] (Fig. 10) to obtain the positive sequence filter

$$
\mathbf{Q}_{S O G I}(s)=\frac{k \omega_{h}^{\prime}}{2} \frac{s+j \omega_{h}^{\prime}}{s^{2}+k \omega_{h}^{\prime} s+\omega_{h}^{\prime 2}},
$$

or the negative sequence filter

$$
\mathbf{Q}_{S O G I}^{*}\left(s^{*}\right)=\frac{k \omega_{h}^{\prime}}{2} \frac{s-j \omega_{h}^{\prime}}{s^{2}+k \omega_{h}^{\prime} s+\omega_{h}^{\prime 2}} .
$$

Transfer functions (2) and (3) have complex coefficients and are thus complex filters ${ }^{1}$ which for $\omega_{h}^{\prime}>0$ provide a damped bandpass response for a positive and negative frequency complex signal respectively. A more direct approach to complex bandpass filtering, however, is through the first-order $\mathrm{CBF}$

$$
\mathbf{Q}(s)=\frac{\mathbf{v}(s)}{\mathbf{u}(s)}=\frac{\omega_{b}}{s-j \omega_{h}^{\prime}+\omega_{b}},
$$

where $\omega_{b}$ and $\omega_{h}^{\prime}$ determine the bandwidth and center frequency of the CBF respectively. A block diagram corresponding to (4) is shown in Fig. 1. The CBF has a settling time defined by $\tau_{b}=5 / \omega_{b}$. Conversely, $\mathbf{Q}_{S O G I}(s)$ has a settling time $\tau_{b, S O G I} \approx 10 /\left(k \omega_{h}^{\prime}\right)$ [2]. This highlights a subtle but important advantage of $\mathbf{Q}(s)$ over $\mathbf{Q}_{S O G I}(s) ; \omega_{h}^{\prime}$ can assume any real number for $\mathbf{Q}(s)$ but is constrained to be positive

\footnotetext{
${ }^{1}$ In complex filtering the notions of gain and phase shift refer to the steady-state relationship between input and output complex exponentials. For an introduction to complex filtering see [9].
}

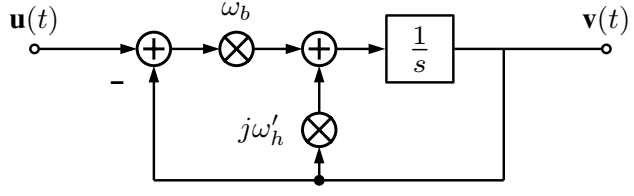

Fig. 1: Block diagram of the continuous $\operatorname{CBF} \mathbf{Q}(s)$.
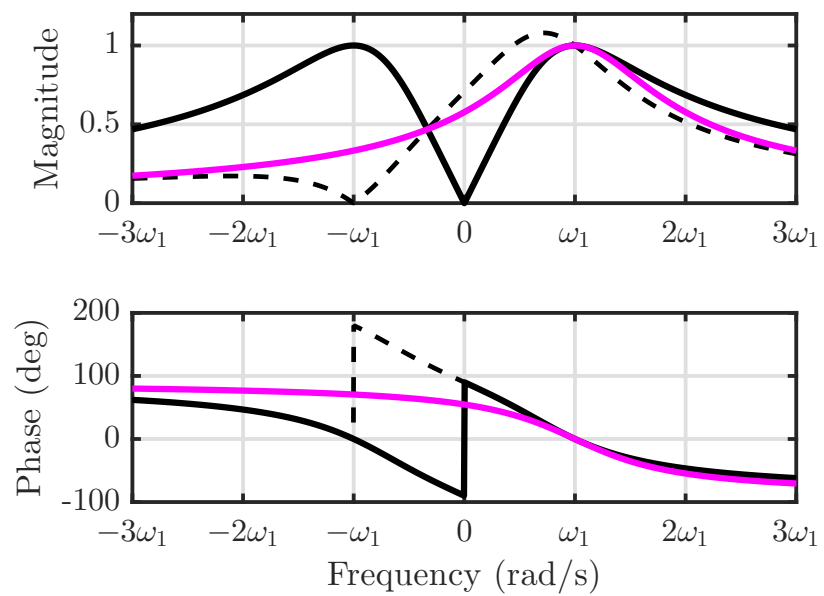

Fig. 2: Frequency response for $Q_{S O G I}(s)(-), \mathbf{Q}_{S O G I}(s)$ $(--)$ and $\mathbf{Q}(s)(-)$ when $\omega_{h}^{\prime}=\omega_{1}, k=\sqrt{2}$ and $\omega_{b}=$ $k\left|\omega_{h}^{\prime}\right| / 2$.

for $\mathbf{Q}_{S O G I}(s)$. Moreover, comparing (4) with (2) shows that the $\mathrm{CBF}$ requires half the number of states compared to the SOGI-based complex filter [13]. A comparison of $Q_{S O G I}(s)$, $\mathbf{Q}_{S O G I}(s)$ and $\mathbf{Q}(s)$ is shown in Fig. 2 when $\omega_{h}^{\prime}=\omega_{1}$, $\omega_{b}=k\left|\omega_{h}^{\prime}\right| / 2$ and $k=\sqrt{2}$ (to achieve a critically damped response [1]). By inspection $\mathbf{Q}(s)$ has the same asymptotic properties as $\mathbf{Q}_{S O G I}(s)$ as $\omega \rightarrow \infty$ but has a symmetrical magnitude response around $\omega=\omega_{1}$.

\section{B. Design of a Discrete Complex Bandpass Filter}

A variety of techniques exist for obtaining discrete filters [24],[25]. The continuous second-order bandpass filters reported in [1]-[3],[5]-[8] invariably require an approximation of the continuous integrators for the digital implementation. The discrete implementation of the continuous CBF reported in [11],[12] and the complex pre-filters in [17] encouraged using a 3rd-order approximation of the continuous integrator

$$
\frac{1}{s} \approx \frac{T_{s}}{12} \frac{23 z^{-1}-16 z^{-2}+5 z^{-3}}{1-z^{-1}},
$$

where $T_{s}$ is the sampling time of the digital system. Let the discrete filters obtained by applying (5) to (2) and (4) be denoted $\mathbf{Q}_{\mathrm{SOGI}, 3 r d}(z)$ and $\mathbf{Q}_{3 \mathrm{rd}}(z)$ respectively. The number of real-valued operations and memory elements for $\mathbf{Q}_{\text {SOGI,3rd }}(z)$ and $\mathbf{Q}_{3 \mathrm{rd}}(z)$ are compared in Table I (recall that one complex multiplication requires four real multiplications and two real additions). Not surprisingly, $\mathbf{Q}_{3 \mathrm{rd}}(z)$ requires significantly fewer operations and memory elements than $\mathbf{Q}_{\mathrm{SOGI}, 3 \mathrm{rd}}(z)$, which supports the CBF-based approach for filtering in threephase systems [12]. 


\begin{tabular}{lccc}
\hline Complex Filter & Additions & Multiplications & Memory \\
\hline $\mathbf{Q}_{\text {SOGI,3rd }}(z)$ (Fig. 10 in [1]) & 18 & 24 & 12 \\
$\mathbf{Q}_{3 \text { rd }}(z)$ & 10 & 14 & 6 \\
$\mathbf{Q}(z)$ & 4 & 8 & 2 \\
\hline
\end{tabular}

TABLE I: Real-valued numerical operations and memory requirements for $\mathbf{Q}_{\text {SOGI,3rd }}(z), \mathbf{Q}_{3 r d}(z)$ and $\mathbf{Q}(z)$.

A more serious issue when approximating integrators, however, is that the resultant bandpass filters are only stable when the center frequency is much smaller than the sampling frequency i.e. $\omega_{h}^{\prime} \ll \omega_{s}$. Consider a practical high-power converter with sampling frequency $f_{s}=5 \mathrm{kHz}$. Let $\omega_{h}^{\prime}=$ $0.1 \omega_{s}$ (i.e. $f_{h}^{\prime}=500 \mathrm{~Hz}$.) and $\tau_{b}=20 \mathrm{~ms}$. The frequency responses for $\mathbf{Q}(s)$ and $\mathbf{Q}_{3 \mathrm{rd}}(z)$ are shown in Fig. 3. Whilst $\mathbf{Q}_{3 \mathrm{rd}}(z)$ is stable, it poorly replicates the continuous filter at $\omega_{h}^{\prime}$ and exhibits anomalous peaks. Furthermore, $\mathbf{Q}_{3 \mathrm{rd}}(z)$ becomes unstable when $\omega_{h}^{\prime} \geq 0.12 \omega_{s}$.

Completing the CBF design in the discrete frequency domain avoids the aforementioned stability issues and allows further reduction of the computational burden. The bandwidth and center frequency of the continuous CBF are mapped to the discrete frequency domain by placing a discrete pole at

$$
z_{\text {pole }}=e^{s_{\text {pole }} T_{s}}=e^{-\left(\omega_{b}-j \omega_{h}^{\prime}\right) T_{s}},
$$

where $s_{\text {pole }}=-\omega_{b}+j \omega_{h}^{\prime}$ is the continuous pole. A first-order discrete $\operatorname{CBF} \mathbf{Q}(z)$ is then obtained by placing a zero at the origin giving

$$
\mathbf{Q}(z)=\frac{\left(1-e^{-\omega_{b} T_{s}}\right) z}{z-e^{-\left(\omega_{b}-j \omega_{h}^{\prime}\right) T_{s}}},
$$

with the magnitude normalized such that $\mathbf{Q}\left(e^{j \omega_{h}^{\prime} T_{s}}\right)=1$. $\mathbf{Q}(z)$ is actually an impulse invariant [24] realization of $\mathbf{Q}(s)$ and has been used in biomedical applications [26]. $\mathbf{Q}(z)$ is stable at all center frequencies and closely replicates the frequency response of the continuous filter as shown in Fig. 3. A direct-form I implementation of $\mathbf{Q}(z)$ is shown in Fig. 4a. The number of real-valued operations and storage elements for $\mathbf{Q}(z)$, noted in Table I, are further reduced compared to $\mathbf{Q}_{3 \mathrm{rd}}(z)$.

It is insightful to compare $\mathbf{Q}(z)$ with other first-order discrete $\mathrm{CBF}$ forms. Consider a discrete $\mathrm{CBF} \mathbf{Q}_{x}(z)$ having the same pole as $\mathbf{Q}(z)$ but with no (finite) zero such that

$$
\mathbf{Q}_{x}(z)=\frac{\left(1-e^{-\omega_{b} T_{s}}\right) e^{j \omega_{h}^{\prime} T_{s}}}{z-e^{-\left(\omega_{b}-j \omega_{h}^{\prime}\right) T_{s}}} .
$$

The discrete filter shown in Fig. 1a of [13] is the same as (8) when written in complex SISO form. In fact, (8) is obtained through a forward integration of the baseband allpass filter

$$
A(s)=\frac{s-\omega_{b}}{s+\omega_{b}},
$$

followed by a frequency shift $z^{-1} \rightarrow e^{j \omega_{h}^{\prime} T_{s}} z^{-1}$, giving the discrete complex allpass filter $\mathbf{A}_{x}(z) . \mathbf{Q}_{x}(z)$ is then obtained as $\mathbf{Q}_{x}(z)=\left(1-\mathbf{A}_{x}(z)\right) / 2$. Forward integration is known to give discrete filters with poorer stability properties (and potential instability) compared to those obtained by other methods [27]. Consider the phase responses of $\mathbf{Q}(z)$ and
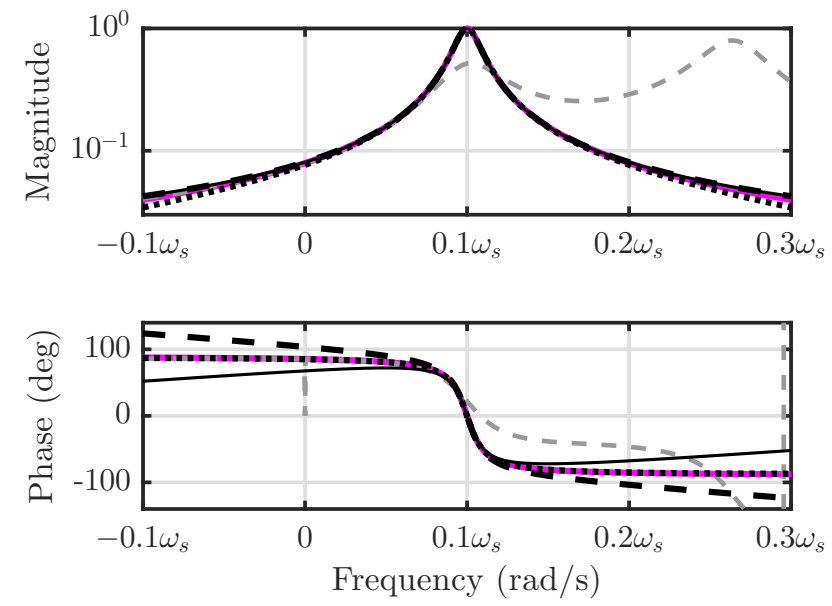

Fig. 3: Frequency response for $\mathbf{Q}(s)(-), \mathbf{Q}_{3 \mathrm{rd}}(z)(=-=)$, $\mathbf{Q}(z)(\boldsymbol{- -}), \mathbf{Q}_{x}(z)(-)$ and $\mathbf{Q}_{y}(z)(\cdots \cdot)$ when $T_{s}=200 \mu s$, $\omega_{h}^{\prime}=0.1 \omega_{s}$ and $\tau_{b}=20 \mathrm{~ms}$.

$\mathbf{Q}_{x}(z)$ in Fig. 3. Note that the phase of $\mathbf{Q}_{x}\left(e^{j \omega T_{s}}\right)$ exceeds $\pm 90^{\circ}$ in the stopband region whereas the phase of $\mathbf{Q}(j \omega)$ and $\mathbf{Q}\left(e^{j \omega T_{s}}\right)$ does not. $\mathbf{Q}_{x}(z)$ is stable, yet the larger phase shift can degrade performance when the filter is used in a closedloop system. A direct-form II implementation of $\mathbf{Q}_{x}(z)$ is given in Fig. 4b. Note that the output always receives delayed information from the input.

A third discrete $\operatorname{CBF} \mathbf{Q}_{y}(z)$ is considered by placing the zero at $z_{z e r o}=-\exp \left(-\left(\omega_{b}-j \omega_{h}^{\prime}\right) T_{s}\right)$ to give the normalized filter

$$
\mathbf{Q}_{y}(z)=\tanh \left(\omega_{b} T_{s} / 2\right) \cdot \frac{z+e^{-\left(\omega_{b}-j \omega_{h}^{\prime}\right) T_{s}}}{z-e^{-\left(\omega_{b}-j \omega_{h}^{\prime}\right) T_{s}}} .
$$

$\mathbf{Q}_{y}(z)$ is actually a corrected impulse invariant [28] realization of $\mathbf{Q}(s)$ and has a digital implementation as a direct-form II filter as shown in Fig. 4c. The magnitude response of $\mathbf{Q}_{y}(z)$, shown in Fig. 3, has slightly better stopband attenuation than $\mathbf{Q}(z)$ and $\mathbf{Q}_{x}(z)$ yet the phase response remains within $\pm 90^{\circ}$.

Out of the discrete forms that have been derived $\mathbf{Q}(z)$ is of most interest because:

1) $\mathbf{Q}(z)$ is stable at all center frequencies when $\omega_{b}>0$.

2) $\mathbf{Q}(z)$ has an improved phase response over $\mathbf{Q}_{x}(z)$.

3) For most purposes the poorer stopband attenuation of $\mathbf{Q}(z)$ compared to $\mathbf{Q}_{y}(z)$ is of negligible concern.

4) The delayed output, $\exp \left(j \omega_{h}^{\prime} T_{s}\right) \mathbf{v}(n-1)$, is inherently available in $\mathbf{Q}(z)$, facilitating causal decoupling networks without requiring extra calculations or storage.

Accordingly, $\mathbf{Q}(z)$ is the recommended first-order discrete CBF form and is exclusively used in the rest of this paper (unless otherwise stated). Note that the analytic functions $\exp \left(-\omega_{b} T_{s}\right), \exp \left(j \omega_{h}^{\prime} T_{s}\right)$ and $\tanh \left(\omega_{b} T_{s} / 2\right)$ can be approximated by truncated Maclaurin series to reduce computational burden. Particular care should be taken to ensure the correct pole placement. This type of analysis proceeds along the same lines as proposed in [29] for the cosine function. 


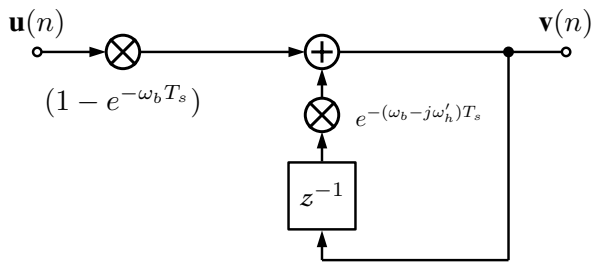

(a) $\mathbf{Q}(z)$ implemented as a direct-form I filter.

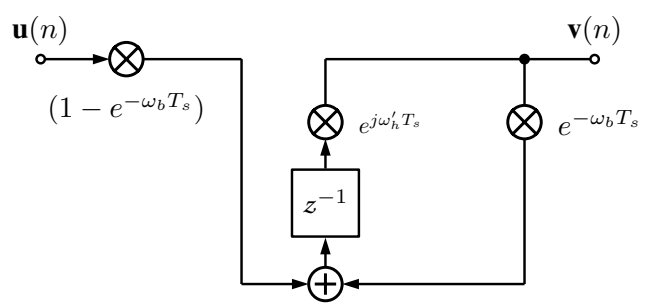

(b) $\mathbf{Q}_{x}(z)$ implemented as a direct-form II filter.

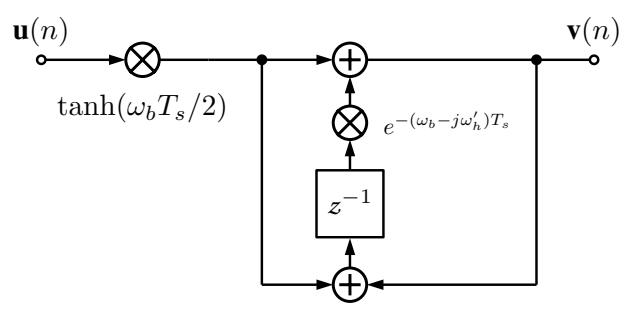

(c) $\mathbf{Q}_{y}(z)$ implemented as a direct-form II filter.

Fig. 4: Digital implementations of $\mathbf{Q}(z), \mathbf{Q}_{x}(z)$ and $\mathbf{Q}_{y}(z)$ with one complex delay element.

\section{Higher-Order Complex Bandpass Filters}

Higher-order filters can be obtained by cascading lowerorder filters and offer reduced settling times and/or high stopband attenuation [30]. In most cases going from a firstorder filter to a second-order filter brings a large improvement in stopband attenuation with increasingly higher-order filters offering diminishing returns [17]. Therefore, a second-order discrete CBF is of interest.

A pre-filtered positive sequence filter was proposed in Fig. 16 of [23], giving the transfer function

$$
\mathbf{Q}_{\text {SOGI }}(s)=Q_{S O G I}(s) \mathbf{Q}_{S O G I}(s),
$$

where $Q_{S O G I}(s)$ and $\mathbf{Q}_{S O G I}(s)$ are (1) and (2) respectively. Interestingly, using $Q_{S O G I}(s)$ as the pre-filter in $\mathbf{Q}_{S O G I 2}(s)$ creates a notch in the frequency response at the zero frequency, which can be advantageous for attenuating very low frequency signals. Alternatively, a $p$ th-order positive sequence filter can be obtained by simply cascading $\mathbf{Q}_{S O G I}(s) p$ times as

$$
\mathbf{Q}_{S O G I}^{p}(s)=\left(\frac{k_{p} \omega_{h}^{\prime}}{2} \frac{s+j \omega_{h}^{\prime}}{s^{2}+k_{p} \omega_{h}^{\prime} s+\omega_{h}^{\prime 2}}\right)^{p} .
$$

Similarly, a $p$ th-order CBF is obtained by cascading the firstorder CBF $\mathbf{Q}(s) p$ times, giving repeated poles at $s=-\omega_{b p}+$ $j \omega_{h}^{\prime}$ to enhance stopband attenuation:

$$
\mathbf{Q}^{p}(s)=\frac{\omega_{b p}^{p}}{\left(s+\omega_{b p}-j \omega_{h}^{\prime}\right)^{p}}
$$

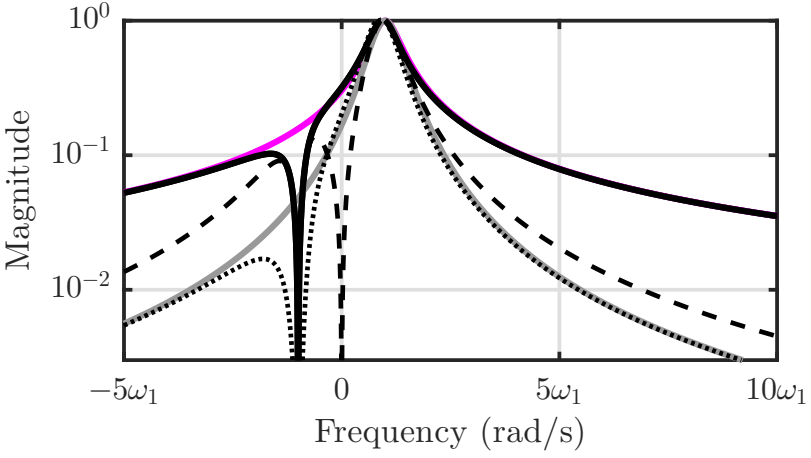

Fig. 5: Magnitude responses of $\mathbf{Q}_{S O G I}(s)(-), \mathbf{Q}(s)(-)$, $\mathbf{Q}_{S O G I}^{2}(s)(\cdots \cdots), \mathbf{Q}^{2}(s)(-)$ and $\left.\mathbf{Q}_{S O G I 2}(s)(--)\right)$ when $\tau_{b}=\tau_{b, S O G I}=50 \mathrm{~ms}$ and $\omega_{h}^{\prime}=\omega_{1}$.

The bandwidth of $\mathbf{Q}^{p}(s), \omega_{b p}$, is normalized to $\mathbf{Q}(s)$ by setting

$$
\omega_{b p}=\sqrt{2}^{p-1} \omega_{b}
$$

Similarly, the damping coefficient used in $\mathbf{Q}_{S O G I}^{p}(s)$ is normalized as $k_{p}=\sqrt{2}^{p-1} k$. Subject to these normalizations and when $p \leq 3$ the settling times of $\mathbf{Q}^{p}(s)$ and $\mathbf{Q}_{S O G I}^{p}(s)$ will be approximately $\tau_{b}=\sqrt{2}^{p-1} 5 / \omega_{b p}$ and $\tau_{b, S O G I}=$ $\sqrt{2}^{p-1} 10 /\left(k_{p} \omega_{h}^{\prime}\right)$ respectively. A comparison of $\mathbf{Q}_{S O G I}(s)$, $\mathbf{Q}(s), \mathbf{Q}_{S O G I}^{2}(s), \mathbf{Q}^{2}(s)$ and $\mathbf{Q}_{S O G I 2}(s)$ is given in Fig. 5 when $\tau_{b}=\tau_{b, S O G I}=50 \mathrm{~ms}$ and $\omega_{h}^{\prime}=\omega_{1}$. The magnitude response of $\mathbf{Q}_{\text {SOGI2 }}(s)$ around $-\omega_{1}$ has poorer attenuation than $\mathbf{Q}_{S O G I}^{2}(s)$ justifying (13) instead of (12) as a positive sequence extractor based on cascaded SOGIs. The magnitude response of $\mathbf{Q}^{2}(s)$ closely matches that of $\mathbf{Q}_{S O G I}^{2}(s)$, indicating that a second-order CBF essentially provides the same stopband attenuation as cross-coupled fourth-order real filters.

Similar to the continuous case, a $p$ th-order discrete CBF is obtained by cascading $\mathbf{Q}(z) p$ times to give

$$
\mathbf{Q}^{p}(z)=\frac{\left(1-e^{-\omega_{b p} T_{s}}\right)^{p}}{\left(1-e^{-\left(\omega_{b p}-j \omega_{h}^{\prime}\right) T_{s}} z^{-1}\right)^{p}} .
$$

The $p$ th-order discrete CBF is stable as the repeated poles reside inside the unit circle. Fig. 6 shows $\mathbf{Q}(z)$ and $\mathbf{Q}^{2}(z)$ compared to the corresponding continuous CBFs when $T_{s}=$ $200 \mu s, \omega_{h}^{\prime}=0.1 \omega_{s}$ and $\tau_{b}=20 \mathrm{~ms}$. The larger phase-shift of $\mathbf{Q}^{2}\left(e^{j \omega T_{s}}\right)$ is a result of the repeated pole and should be considered when used in closed-loop control.

\section{Frequency AdAPtion for Complex Signals}

Frequency-adaption through an FLL allows the center frequency of the CBF to be updated with an estimation of the largest frequency component in the input signal [30]. Frequency adaption is a highly nonlinear process [13], with analysis methods based on continuous state variables [2] and $d q$ variable transformation [13]. Alternatively, Regalia [19] analyses the cross-spectrum of the adaption mechanism to find the expected (average) evolution of the frequency estimation of a discrete complex ANF. This method is tractable as the influence of noise and the bandpass filter structure on the FLL performance becomes apparent and complicated filter structures can be readily analyzed. Regalia's approach is used 

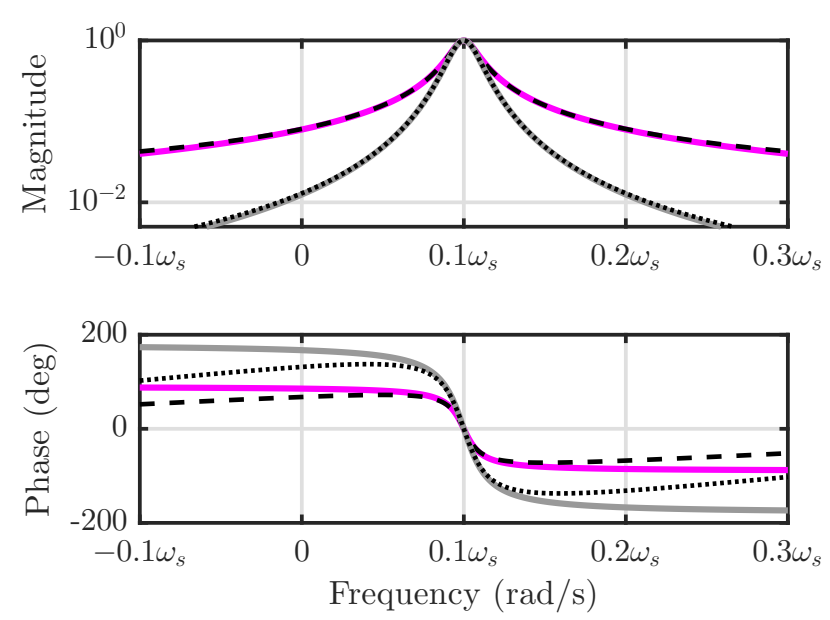

Fig. 6: Frequency response for $\mathbf{Q}(s)(-), \mathbf{Q}(z)(---), \mathbf{Q}^{2}(s)$ $(-)$ and $\mathbf{Q}^{2}(z)(\cdots \cdots)$ when $T_{s}=200 \mu s, \omega_{h}^{\prime}=0.1 \omega_{s}$ and $\tau_{b}=20 \mathrm{~ms}$.

in this paper to develop a normalized FLL for the $p$ th-order discrete CBF.

Normalization of the FLL has the advantage that the frequency-adaption becomes approximately independent of the magnitude of the input signal [2],[13]. Furthermore, this section will show how the tuning of the FLL can be made approximately independent of the discrete CBF form. This allows the stability of the FLL to be approximately defined by the range of the tuning parameter $0<\gamma T_{s}<1$.

\section{A. Discrete-Time Frequency-Adaption}

A block diagram of the normalized FLL is shown in Fig. $7 \mathrm{a}$ and is described by the difference equation

$$
\begin{aligned}
\omega_{h}^{\prime}(n+1) & =\omega_{h}^{\prime}(n)-\gamma K \frac{\left(v_{\beta}(n) w_{\alpha}(n)-v_{\alpha}(n) w_{\beta}(n)\right)}{v_{\alpha}(n)^{2}+v_{\beta}(n)^{2}} \\
& =\omega_{h}^{\prime}(n)-\gamma K \cdot \operatorname{Im}\left\{\frac{\mathbf{v}(n) \mathbf{w}^{*}(n)}{|\mathbf{v}(n)|^{2}}\right\}
\end{aligned}
$$

where $n$ is the sample number, $\gamma$ controls the adaption rate and $K$ is a normalizing constant that will be determined shortly. As shown in Fig. 7b, $\mathbf{v}(n)=v_{\alpha}(n)+j v_{\beta}(n)$ is the output of the $p$ th-order discrete $\mathrm{CBF}$ and $\mathbf{w}(n)=w_{\alpha}(n)+j w_{\beta}(n)$ is the output of the $(p-1)$ th cascaded first-order section $\left(\omega_{b p}\right.$ is the cut-off frequency for all sections). This configuration gives the $p$ th-order CBF-FLL an improved frequency estimation over the first-order CBF-FLL as both input $\mathbf{v}(n)$ and $\mathbf{w}(n)$ are bandpass filtered signals (if $p=1$ then $\mathbf{w}(n)=\mathbf{u}(n)$, which is unfiltered).

As described in [19], for sufficiently slow frequency adaption the FLL can be modeled by its expected evolution

$$
\bar{\omega}_{h}^{\prime}(n+1)=\bar{\omega}_{h}^{\prime}(n)-\gamma K \cdot \operatorname{Im}\left\{E\left[\frac{\mathbf{v}(n) \mathbf{w}^{*}(n)}{|\mathbf{v}(n)|^{2}}\right]\right\},
$$

where the bar notation replaces the expectation operator. To avoid numerical saturation the expectation on the right hand side of (17) must be restricted to

$$
E\left[\frac{\mathbf{v}(n) \mathbf{w}^{*}(n)}{|\mathbf{v}(n)|^{2}}\right] \text { subject to }|\mathbf{v}(n)|^{2} \neq 0 .
$$

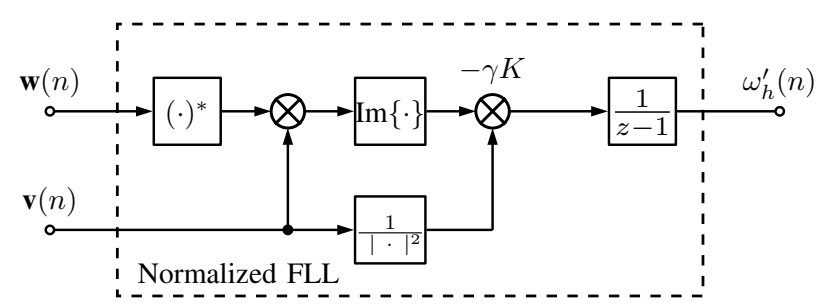

(a) Normalized frequency locked-loop (FLL).

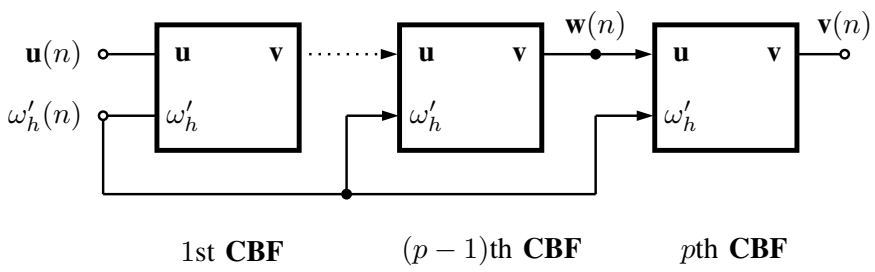

(b) $p$ th-order discrete CBF comprised of first-order sections given by $\mathbf{Q}(z)$ with bandwidth $\omega_{b p}$.

Fig. 7: Connection diagram for achieving frequency-adaption in a $p$ th-order discrete CBF comprised of $p$ first-order sections.

A bivariate Taylor expansion of (18) at the point $\left(E\left[\mathbf{v}(n) \mathbf{u}^{*}(n)\right], E\left[|\mathbf{v}(n)|^{2}\right]\right)$ gives

$$
E\left[\frac{\mathbf{v}(n) \mathbf{w}^{*}(n)}{|\mathbf{v}(n)|^{2}}\right]=\frac{E\left[\mathbf{v}(n) \mathbf{w}^{*}(n)\right]}{E\left[|\mathbf{v}(n)|^{2}\right]}+\text { HOTs, }
$$

with the higher-order terms (HOTs) noted in [31]. The subsequent analysis proceeds without the HOTs, amounting to a first-order approximation of (18).

Let the input signal $\mathbf{u}(n)=u_{\alpha}(n)+j u_{\beta}(n)$ be a complex exponential of amplitude $V$, frequency $\omega_{h}$ and phase $\theta$, immersed in zero-mean complex circular white noise $\mathbf{b}(n)$ with variance $\sigma^{2}$ [19] such that

$$
\mathbf{u}(n)=V e^{j\left(\omega_{h} n+\theta\right)}+\mathbf{b}(n) .
$$

The power spectral density of $\mathbf{u}(n)$ is then

$$
S_{u u}(\omega)=2 \pi V^{2} \delta\left(\omega-\omega_{h}\right)+\sigma^{2},
$$

where $\delta\left(\omega-\omega_{h}\right)$ is the Kronecker-delta function centered at $\omega=\omega_{h} . E\left[\mathbf{v}(n) \mathbf{w}^{*}(n)\right]$ is a cross-covariance with zero lag and relates to the input power spectral density through an inverse Fourier transform of the output power spectral density [32]:

$$
\begin{aligned}
E\left[\mathbf{v}(n) \mathbf{w}^{*}(n)\right] & =\frac{1}{2 \pi} \int_{-\pi}^{\pi} S_{v w}(\omega) d \omega \\
& =\frac{1}{2 \pi} \int_{-\pi}^{\pi} \mathbf{Q}^{p}\left(e^{j \omega}\right) \mathbf{Q}^{(p-1) *}\left(e^{j \omega}\right) S_{u u}(\omega) d \omega \\
& =V^{2} \frac{\left|\mathbf{Q}^{p}\left(e^{j \omega_{h}}\right)\right|^{2}}{\mathbf{Q}^{*}\left(e^{j \omega_{h}}\right)}+\frac{\sigma^{2}}{2 \pi} \int_{-\pi}^{\pi} \frac{\left|\mathbf{Q}^{p}\left(e^{j \omega}\right)\right|^{2}}{\mathbf{Q}^{*}\left(e^{j \omega}\right)} d \omega
\end{aligned}
$$


Similarly, $E\left[|\mathbf{v}(n)|^{2}\right]$ is an auto-covariance and relates to the input power spectral density as

$$
\begin{aligned}
E\left[|\mathbf{v}(n)|^{2}\right] & =\frac{1}{2 \pi} \int_{-\pi}^{\pi} S_{v v}(\omega) d \omega \\
& =\frac{1}{2 \pi} \int_{-\pi}^{\pi} \mathbf{Q}^{p}\left(e^{j \omega}\right) \mathbf{Q}^{p *}\left(e^{j \omega}\right) S_{u u}(\omega) d \omega \\
& =V^{2}\left|\mathbf{Q}^{p}\left(e^{j \omega_{h}}\right)\right|^{2}+\frac{\sigma^{2}}{2 \pi} \int_{-\pi}^{\pi}\left|\mathbf{Q}^{p}\left(e^{j \omega}\right)\right|^{2} d \omega .
\end{aligned}
$$

According to (23), normalization allows the noise variance to enter the FLL dynamics as the integrand is a positive-valued function for $-\pi \leq \omega \leq \pi$. However, provided the signal-tonoise ratio is high, $\sigma^{2} /\left(2 V^{2}\right) \approx 0$ such that

$$
\frac{E\left[\mathbf{v}(n) \mathbf{w}^{*}(n)\right]}{E\left[|\mathbf{v}(n)|^{2}\right]}=1 / \mathbf{Q}^{*}\left(e^{j \omega_{h}}\right)=\frac{1-e^{-\omega_{b p} T_{s}} e^{-j\left(\bar{\omega}_{h}^{\prime}-\omega_{h}\right)}}{1-e^{-\omega_{b p} T_{s}}},
$$

where $\omega_{b p}=\sqrt{2}^{p-1} \omega_{b}$ as noted in (14). The imaginary part of (24) is then

$$
\operatorname{Im}\left\{\frac{E\left[\mathbf{v}(n) \mathbf{w}^{*}(n)\right]}{E\left[|\mathbf{v}(n)|^{2}\right]}\right\}=\frac{e^{-\omega_{b p} T_{s}}}{\left(1-e^{-\omega_{b p} T_{s}}\right)} \sin \left(\bar{\omega}_{h}^{\prime}-\omega_{h}\right) .
$$

Applying the approximation of (25) to (17) and letting $\sin \left(\bar{\omega}_{h}^{\prime}-\omega_{h}\right) \approx \bar{\omega}_{h}^{\prime}-\omega_{h}$ gives

$$
\bar{\omega}_{h}^{\prime}(n+1) \approx \bar{\omega}_{h}^{\prime}(n)-\gamma K \cdot \frac{e^{-\omega_{b p} T_{s}}}{1-e^{-\omega_{b p} T_{s}}}\left(\bar{\omega}_{h}^{\prime}-\omega_{h}\right),
$$

suggesting a normalization constant of

$$
K=\frac{1-e^{-\omega_{b p} T_{s}}}{e^{-\omega_{b p} T_{s}}} .
$$

The normalization constant $K$ is dependent on the discrete form of the $\mathrm{CBF}$. Other forms will require a different scaling to achieve a normalized FLL, but can be derived using the same approach. Substituting (27) into (26) and assuming $\omega_{h}(n)$ is slowly time-varying gives the following approximated firstorder difference equation

$$
\bar{\omega}_{h}^{\prime}(n+1) \approx(1-\gamma) \bar{\omega}_{h}^{\prime}(n)+\gamma \omega_{h}(n) .
$$

In continuous time $\gamma$ is scaled to $\gamma T_{s}$. Accordingly, the Final Value Theorem ensures that, for a step change in $\omega_{h}(n)$, $\omega_{h}^{\prime}(n)$ converges monotonically to $\omega_{h}(n)$ with an approximate settling time of $\tau_{\gamma}=5 / \gamma$, if $0<\gamma T_{s}<1$. The minimum settling time for stable FLL operation is then approximately $\tau_{\gamma, \min }=5 T_{s}$. In comparison, the corresponding unnormalized FLL has a minimum settling time of $\tau_{\gamma, \min } / K$, which is at least ten times larger than for the corresponding normalized FLL, in most practical applications. Note that convergence of $\omega_{h}^{\prime}(n)$ to $\omega_{h}(n)$ is achieved regardless of the polarity of $\omega_{h}(n)$, as required for complex signal processing.

In practice the lower limit in the settling time, $\tau_{\gamma, \text { min }}=$ $5 T_{s}$, should be increased due to the approximations made in the modeling process and due to the presence of harmonics and unknown noise sources. The analysis of the frequency adaption can be extended to include multiple sinusoidal inputs as covered in [30].

\begin{tabular}{cc}
\hline Harmonic Order & Value [pu] \\
\hline 1 & 1 \\
$-5,7,-11,13,-29,31$ & 0.033 \\
\hline
\end{tabular}

TABLE II: Input $\mathbf{u}(n)$ for testing the performance of the discrete CBFs.

\section{EXPERIMENTAL REsults}

The performance improvement of higher-order CBF-FLLs over their first-order counterpart was tested in the form of two industrial applications, voltage synchronization during a grid fault and unknown frequency extraction from a power system signal.

The following results were implemented on a National Instruments cRIO $40 \mathrm{MHz}$ FPGA in floating-point. The CBFFLL algorithms were executed on a fixed $T_{s}=200 \mu \mathrm{s}$ timed loop, replicating a typical sampling period for a high-power converter. A signal generator was used to create the threephase input signal and connected to the input card of the FPGA. The input and output signals of the CBF-FLLs were sampled at $50 \mathrm{kHz}$ for plotting. The fundamental frequency used for the experiments was $\omega_{1}=2 \pi \cdot 50 \mathrm{rad} / \mathrm{s}$.

\section{A. Performance of the pth-order Discrete CBF}

First-, second- and third-order discrete CBFs, $\mathbf{Q}(z), \mathbf{Q}^{2}(z)$ and $\mathbf{Q}^{3}(z)$ respectively, and the approximated continuous $\mathrm{CBF} \mathbf{Q}_{3 \mathrm{rd}}(z)$ were tested for the input signal with harmonics described in Table II. A time-domain plot of the input signal is given in Fig. 8a. The outputs of the CBFs were recorded for center frequencies set to $\omega_{h}^{\prime}=\omega_{1}, \omega_{h}^{\prime}=13 \omega_{1}$ and $\omega_{h}^{\prime}=-29 \omega_{1}$ respectively. Note that $29 \omega_{1}=0.29 \omega_{s}$, represented a significant proportion of the sampling frequency. The settling time of the CBFs was set to $\tau_{b}=5 / \omega_{b}=50 \mathrm{~ms}$.

For brevity only the magnitude of the CBF outputs are given, as shown in Fig. 8b. The response for $\left|\mathbf{v}_{1}(n)\right|$ highlights that all CBFs have the same approximate settling time, verifying the bandwidth normalization given in (14). The approximated continuous filter $\mathbf{Q}_{3 \mathrm{rd}}(z)$ has precisely the same response as the first-order discrete $\operatorname{CBF} \mathbf{Q}(z)$, which is expected as $\mathbf{Q}_{3 \mathrm{rd}}(z)$ is stable for that center frequency. However, the response for $\left|\mathbf{v}_{13}(n)\right|$ highlights that $\mathbf{Q}_{3 \mathrm{rd}}(z)$ immediately diverges due to its unstable pole at that center frequency. A stark difference in the distortion of the responses for $\mathbf{Q}(z)$ and $\mathbf{Q}^{2}(z)$ is also evident, verifying that the secondorder CBF provides significantly improved stopband attenuation over the first-order $\mathrm{CBF} . \mathbf{Q}^{3}(z)$ further improved the stopband attenuation over $\mathbf{Q}^{2}(z)$. Finally, $\mathbf{Q}(z), \mathbf{Q}^{2}(z)$ and $\mathbf{Q}^{3}(z)$ successfully extracted the -29 th harmonic, as shown in $\left|\mathbf{v}_{-29}(n)\right|$, highlighting their stability when centered at high frequencies relative to the sampling frequency.

\section{B. Voltage Synchronization During a Fault}

The application of the $p$ th-order discrete CBF-FLL in voltage synchronization during a fault was tested. Faults can significantly reduce the fundamental positive sequence voltage, introduce a fundamental negative sequence voltage and result in spurious and unknown harmonic distortion due to saturated 


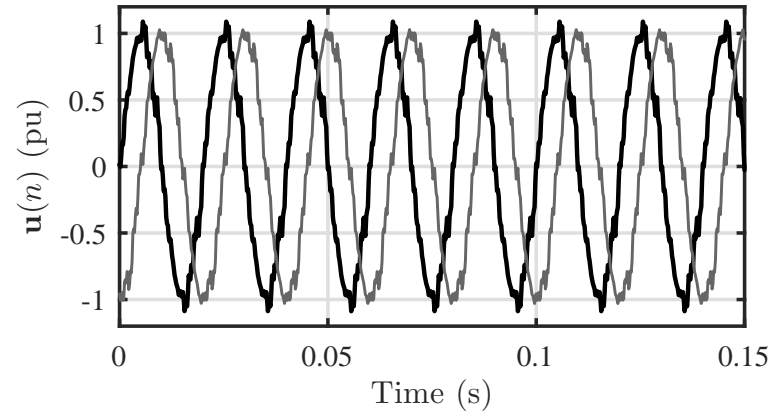

(a) Input signal $\mathbf{u}(n)$ in terms of real and imaginary parts.
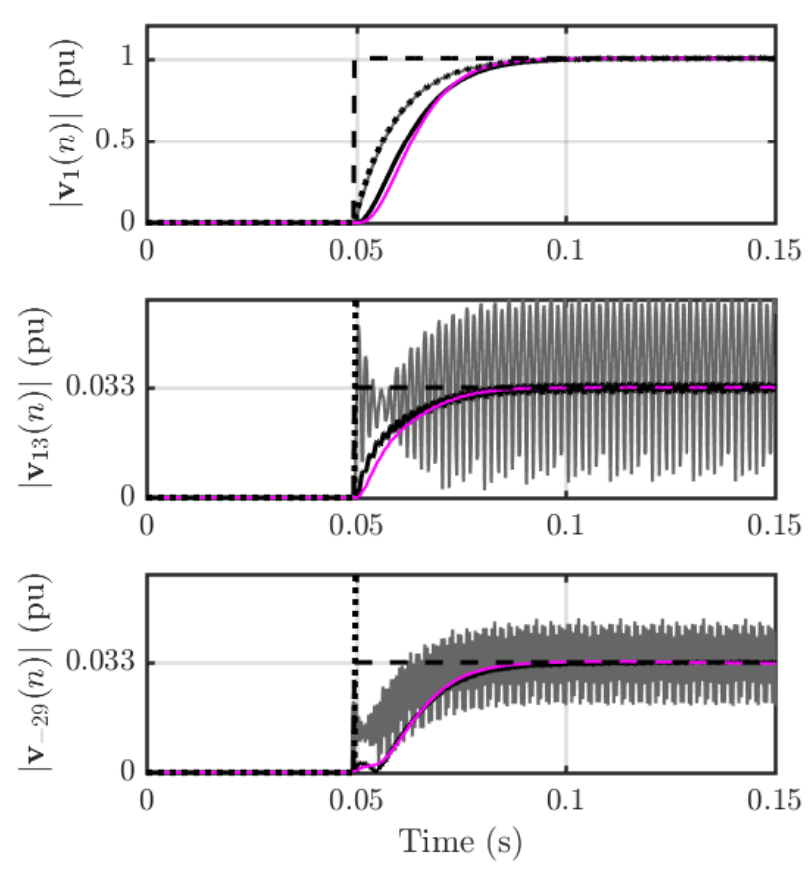

(b) Magnitude of individual CBF outputs for $\mathbf{Q}_{3 \mathrm{rd}}(z)(\ldots \ldots), \mathbf{Q}(z)$ $(-), \mathbf{Q}^{2}(z)(-)$ and $\mathbf{Q}^{3}(z)(-)$. The actual value is given by $(\boldsymbol{-}-\boldsymbol{-})$.

Fig. 8: Testing the performance of the discrete CBFs against the continuous approximation.

transformers. The performance of voltage synchronization during these modes is particularly important in wind turbines where prescribed amounts of active and reactive power must be injected during the fault-ride through window [22]. The fundamental positive and negative sequence voltage should be extracted with minimal distortion to achieve high-performance reference current calculation [33].

Figure 9 shows a CBF-FLL and a CBF connected by a harmonic decoupling network (HDN) [2],[12]. The CBF-FLL estimates the fundamental frequency $\omega_{1}^{\prime}(n)$. The second CBF is tuned to the negative sequence frequency $-\omega_{1}^{\prime}(n)$. The HDN subtracts the output of the CBFs from the inputs of the adjacent CBFs, reducing distortion at the CBF outputs. With firstorder CBFs such a network is effectively the stationary-frame equivalent of the decoupled double synchronous reference frame PLL [17]. Accordingly, using second-order CBFs is of interest due to the improved stopband attenuation of unknown

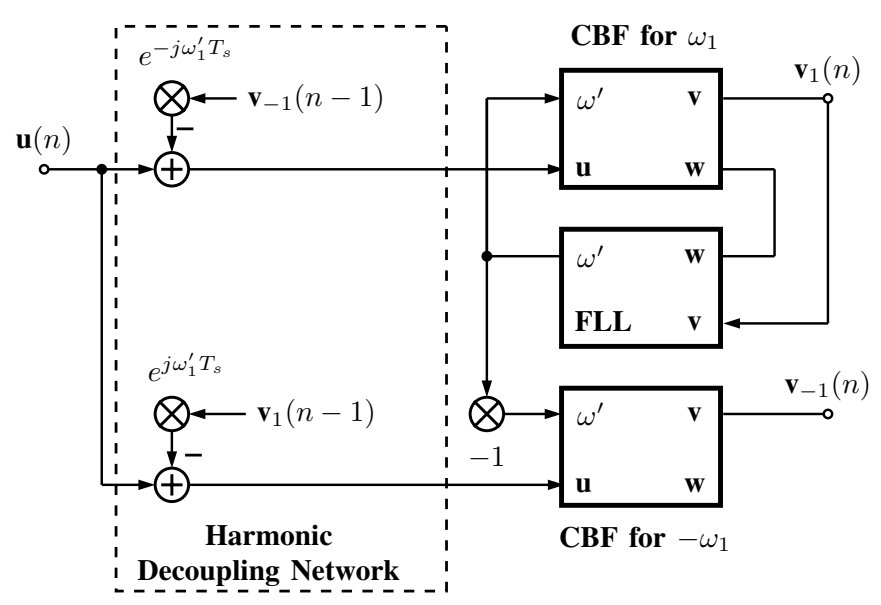

Fig. 9: Two CBFs connected with a HDN for extracting the fundamental positive and negative sequence components.

distortion. Note that the $\mathrm{HDN}$ requires the delayed $\mathrm{CBF}$ outputs to maintain causality. Accordingly, $\mathbf{Q}(z)$ is a desirable choice for the $\mathrm{CBF}$ as the delayed and phase advanced outputs, $\exp \left(j \omega_{1}^{\prime} T_{s}\right) \mathbf{v}_{1}(n-1)$ and $\exp \left(-j \omega_{1}^{\prime} T_{s}\right) \mathbf{v}_{-1}(n-1)$, are inherently available.

The input signal has harmonic content as described in Table III and is shown in Fig. 10a with the emulated fault occurring at $t=0.05 \mathrm{~s}$. After the fault occurs the fundamental frequency steps from $50 \mathrm{~Hz}$ to $45 \mathrm{~Hz}$, emulating a large loss of generation and impressing a step change on the FLL. The settling time of the CBFs were set to $\tau_{b}=5 / \omega_{b}=50 \mathrm{~ms}$ and for the FLLs $\tau_{\gamma}=5 / \gamma=100 \mathrm{~ms}$.

\begin{tabular}{cc}
\hline Harmonic Order & Value $[\mathrm{pu}]$ \\
\hline 1 & 0.2 (1pu pre-fault $)$ \\
-1 & 0.2 \\
$-4,4,-5,7,-11,13$ & 0.2 \\
\hline
\end{tabular}

TABLE III: Input $\mathbf{u}(n)$ for emulating a fault condition.

Figure $10 \mathrm{~b}$ shows that the discrete CBFs respond to the step change in the input signal with the designated settling time. Not surprisingly, the second- and third-order discrete CBFs have significantly improved stopband attenuation over the first-order discrete CBF. The FLL settling time matches the designated value. The second- and third-order CBF-FLLs are better at filtering the distortion in the frequency estimation as both inputs to the FLL, $\mathbf{v}_{1}(n)$ and $\mathbf{w}_{1}(n)$, are bandpass filtered. A closer view of the CBF outputs and the frequency estimation is given in Fig. 11. The improvement in the performance of the second-order CBF-FLL over the first-order CBF-FLL is most notable, extracting the fundamental voltages and frequency with little distortion. These signals can then be passed forward to resonant filters used to regulate current or voltages.

\section{Unknown Frequency Extraction}

The $p$ th-order discrete CBF-FLL was tested in the application of extracting an unknown frequency component from a power system signal. Cascaded adaptive filters can be used to 


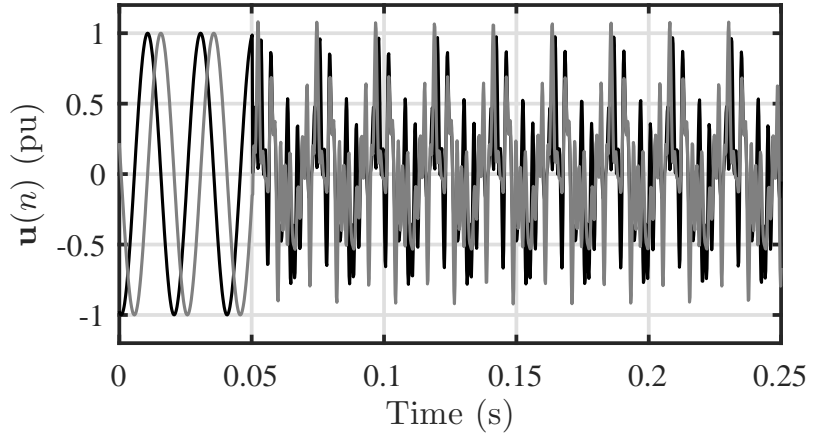

(a) Input signal $\mathbf{u}(n)$ in terms of real and imaginary parts.
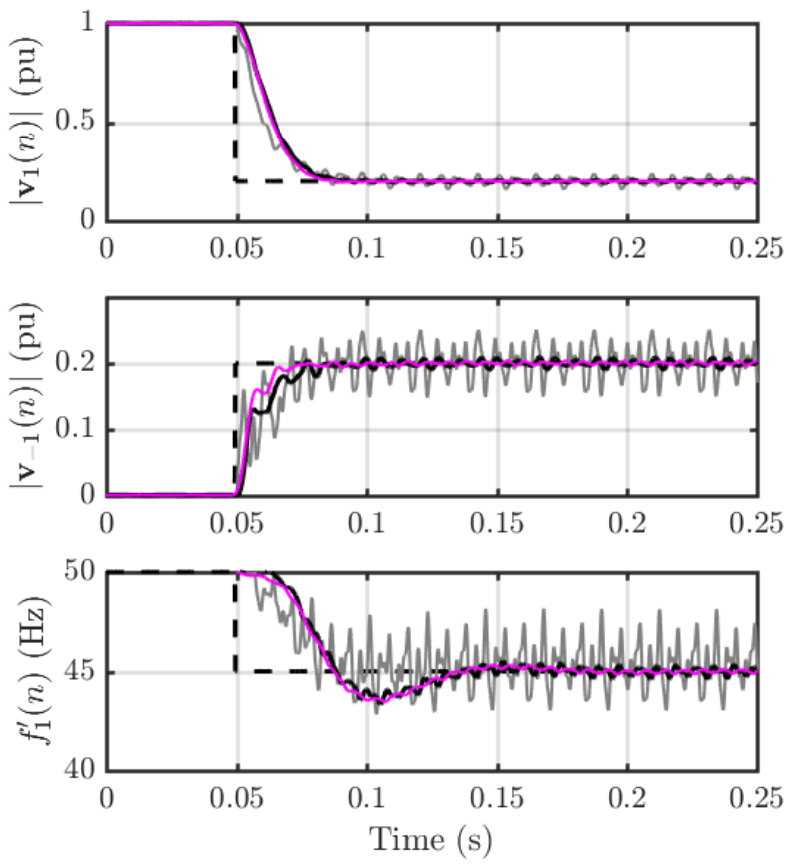

(b) CBF-FLL output magnitude and frequency signals during the emulated fault for $\mathbf{Q}(z)(\square), \mathbf{Q}^{2}(z)(-)$ and $\mathbf{Q}^{3}(z)(\square)$. The actual value is $(\mathbf{-}-\mathbf{-})$.

Fig. 10: Testing of the CBF-FLL when used for voltage synchronization. A fault occurs at $t=0.05 \mathrm{~s}$, causing an unbalanced and distorted input signal.

obtain information about the frequency components with the largest magnitude, but potentially unknown frequency [30]. An advantage of cascaded filter structures over parallel filter structures (such as a HDN) is that deterministic knowledge of the frequency components within the input signal is not strictly required. This is useful for detecting the frequencies of timevarying resonances, and has application in active dampers [21] and in impedance-based active filtering [15],[16]. Moreover, data-driven mechanisms can make use the estimated magnitudes and frequencies for generating trends and diagnostics regarding harmonics and inter-harmonics as well as facilitating parameter adaption [34] for improving converter performance.

Figure 12 shows two cascaded CBF-FLLs for extracting two frequency components from the input signal. The notch filter at the first output separates the frequency component with the largest magnitude at that section (regardless of the polarity
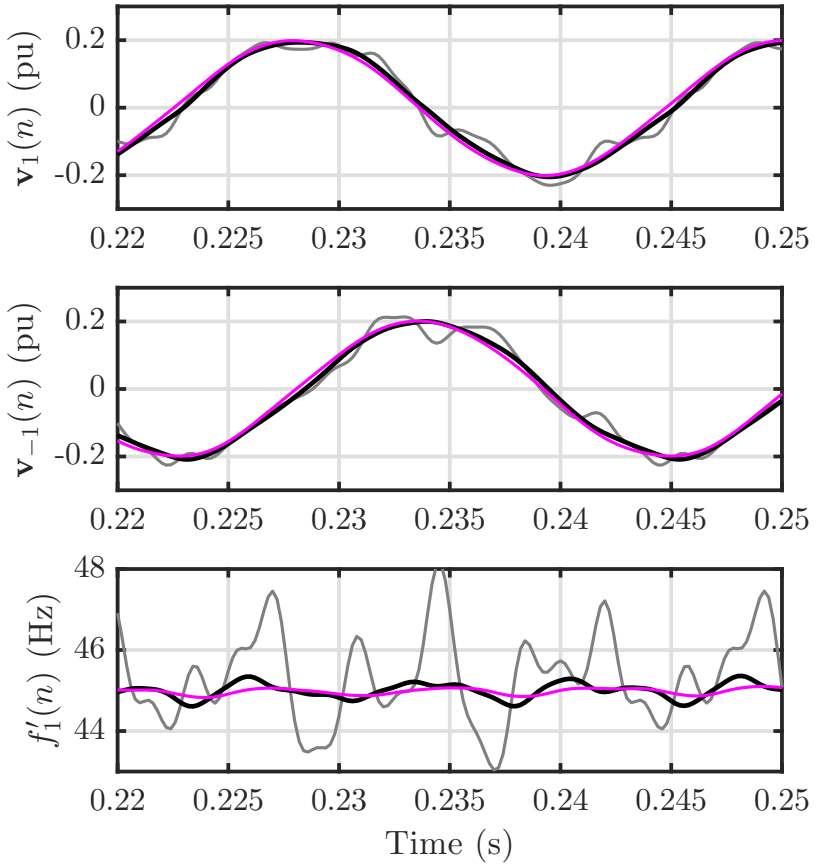

Fig. 11: Close-up of the CBF-FLL signals after the emulated fault has occurred for $\mathbf{Q}(z)(-), \mathbf{Q}^{2}(z)(-)$ and $\mathbf{Q}^{3}(z)$ (-).

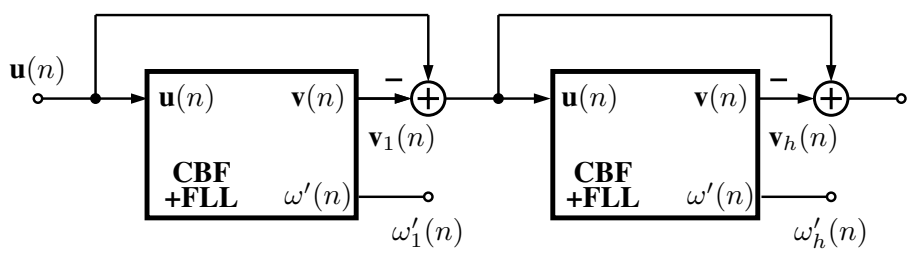

Fig. 12: Two cascaded CBF-FLLs for extracting unknown frequency components with the largest magnitude.

of the frequency). The subsequent CBF-FLL is then forced to seek the remaining frequency components in the signal. The magnitude of the unknown frequency components can differ significantly from the fundamental component. Therefore, normalization is advantageous in ensuring the frequency estimation at each CBF-FLL stage has a defined settling time.

One issue with cascaded filters is that a high filter bandwidth $\omega_{b}$ can allow neighboring frequency components to bias the equilibrium point of a filter section, resulting in incorrect convergence [30]. This issue is managed by reducing the settling time of each $\mathrm{CBF}$ in the cascade, i.e. $\tau_{b, 1}<\tau_{b, 2}<\tau_{b, 3}<\ldots$ where $\tau_{b, i}$ is the settling time for the $i$ th CBF in the cascade of CBF-FLLs.

The input signal used in this experiment is shown in Fig. 13a and contained the harmonic content listed in Table III. The settling time of the first and second CBFs in the cascade were $\tau_{b, 1}=20 \mathrm{~ms}$ and $\tau_{b, 2}=30 \mathrm{~ms}$ respectively. Similarly, the settling time of the first and second FLLs in the cascade were $\tau_{\gamma, 1}=40 \mathrm{~ms}$ and $\tau_{\gamma, 2}=60 \mathrm{~ms}$ respectively. Figures $13 \mathrm{~b}$ and $13 \mathrm{c}$ show the magnitude of the CBF outputs and the frequency estimations respectively. Both the first- and 


\begin{tabular}{cc}
\hline Harmonic Order & Value $[\mathrm{pu}]$ \\
\hline 1 & 1 \\
$-5,7$ & 0.05 \\
$-11,13$ & 0.01 \\
$-12.5 \rightarrow-8.125$ & $0.15 \rightarrow 0.075($ after $t=0.5 s)$ \\
\hline
\end{tabular}

TABLE IV: Input $\mathbf{u}(n)$ for unknown frequency extraction.

\begin{tabular}{lcccc}
\hline Scheme & $\mathbf{Q}_{3 \mathrm{rd}}(z)$ & $\mathbf{Q}(z)$ & $\mathbf{Q}^{2}(z)$ & $\mathbf{Q}^{3}(z)$ \\
\hline Single CBF (4th-Order) & $1.050 \mu \mathrm{s}$ & $1.100 \mu \mathrm{s}$ & $1.375 \mu \mathrm{s}$ & $1.650 \mu \mathrm{s}$ \\
CBF-FLL & - & $2.000 \mu \mathrm{s}$ & $2.275 \mu \mathrm{s}$ & $2.550 \mu \mathrm{s}$ \\
CBF-FLL plus & - & $3.100 \mu \mathrm{s}$ & $3.650 \mu \mathrm{s}$ & $4.200 \mu \mathrm{s}$ \\
CBF (Fig. 9) & - & & & \\
Two Cascaded & - & $4.000 \mu \mathrm{s}$ & $4.550 \mu \mathrm{s}$ & - \\
CBF-FLLs (Fig. 12) & & & & \\
\hline
\end{tabular}

TABLE V: Computational burden for the CBF-FLL schemes.

second-order CBF-FLLs converge at approximately the same rate to the actual values. The main benefit of the secondorder CBF-FLL is the reduced distortion compared to the first-order CBF-FLL. At $t=0.5 \mathrm{~s}$ the resonance changed frequency from $-625 \mathrm{~Hz}$ to $-406.25 \mathrm{~Hz}$. The CBF-FLLs are quickly able to track this frequency change, even bypassing the -11 th harmonic which has appreciable magnitude. This is advantageous as the time-varying resonance frequency can be tracked as the power system changes state. Finally, Fig. 14 shows a close-up of the frequency estimations after the resonance has changed frequency. The second-order CBF-FLL has removed the distortion in the frequency estimation by over $50 \%$, allowing an accurate signal to be passed forward for subsequent current or voltage controllers.

\section{Computational Burden}

The computational burden for the FPGA implementation of the CBFs and FLL is given in Table V. A fourth-order approximation of the cosine and sine functions was used to give a fair comparison between $\mathbf{Q}_{3 \mathrm{rd}}(z)$ and $\mathbf{Q}(z), \mathbf{Q}^{2}(z)$ and $\mathbf{Q}^{3}(z)$. Although $\mathbf{Q}_{3 \mathrm{rd}}(z)$ and $\mathbf{Q}(z)$ have essentially the same computational burden, $\mathbf{Q}(z)$ is preferred as it does not suffer stability issues when the center frequency is increased. Higher-order order approximations of the trig functions can be used when filtering high frequencies relative to the sampling frequencies, costing a marginal increase in computational burden.

The second-order CBF $\mathbf{Q}^{2}(z)$ has a $25 \%$ higher computational burden than $\mathbf{Q}(z)$. When combined with an FLL, however, the relative increase in computational burden of the second-order CBF-FLL over the first-order CBF-FLL is only $13.75 \%$. This is because the FLL is the same regardless of the order of the CBF. Furthermore, based on Figs. 8b, 11 and 14 the second-order CBF-FLL significantly improved both filtering and frequency estimation compared to the first-order CBF-FLL. Accordingly, the second-order CBF-FLL offers a good compromise between performance and computational burden, and is recommended for use in high-performance converter applications.

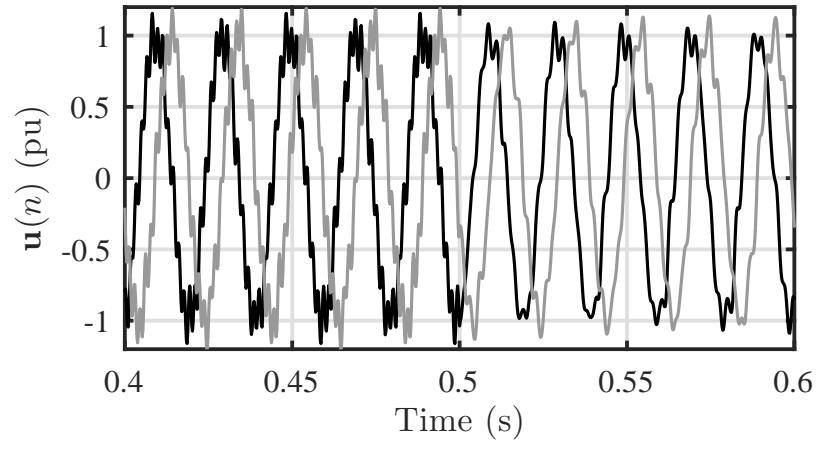

(a) Input signal $\mathbf{u}(n)$ expressed in terms of real and imaginary parts, before and after the resonance changes frequency.
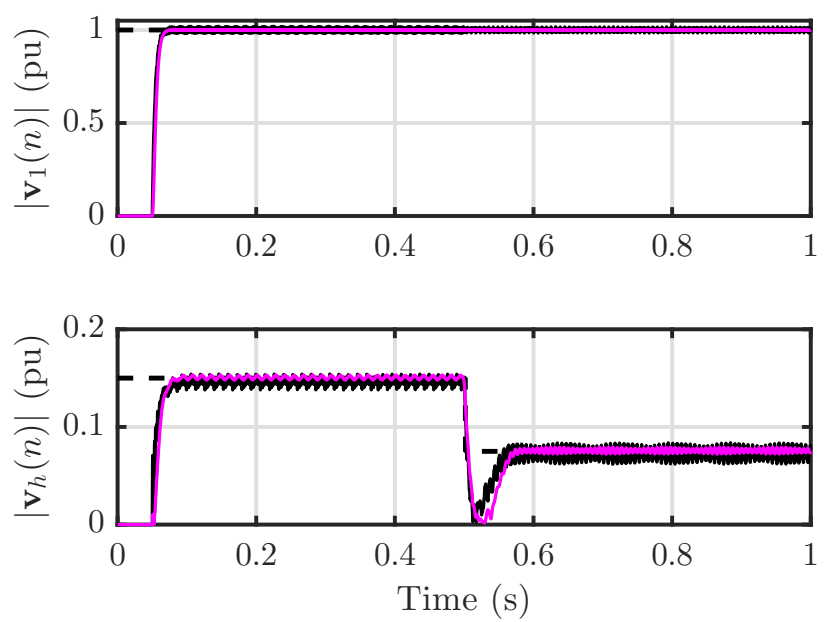

(b) Magnitude of the two cascaded CBF-FLL outputs for $\mathbf{Q}(z)(-)$ and $\mathbf{Q}^{2}(z)(\underline{-})$. The actual value is $(\mathbf{- - -})$.
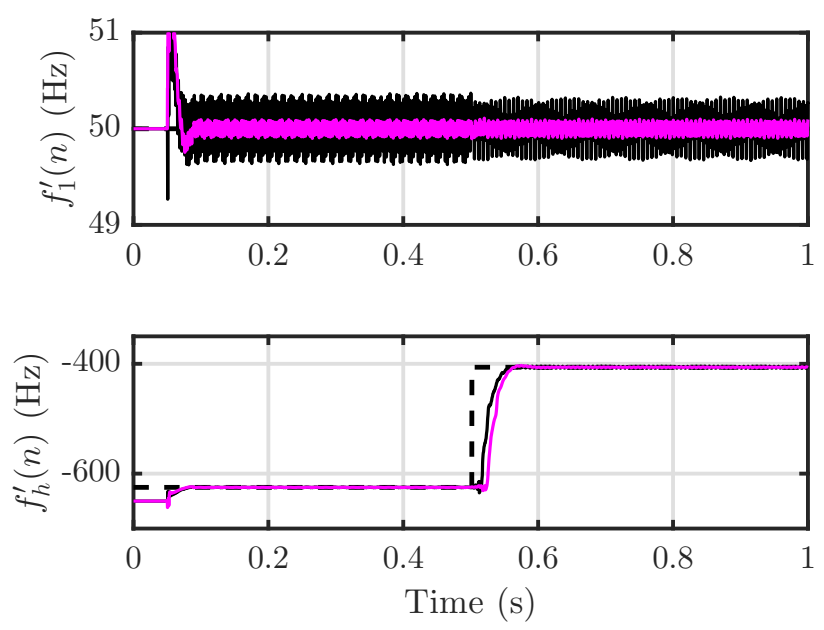

(c) Frequency of the two cascaded CBF-FLLs for $\mathbf{Q}(z)$ (-) and $\mathbf{Q}^{2}(z)(-)$. The actual value is (-- - ) .

Fig. 13: Testing of cascaded CBF-FLLs for extracting an unknown frequency component.

\section{CONCLUSION}

The first-order discrete CBF has been proposed as a building block for complex signal processing units as required in threephase converter applications. The first-order discrete CBF was extended to a $p$ th-order discrete CBF. A normalized FLL was developed for the $p$ th-order discrete CBF with a frequency 

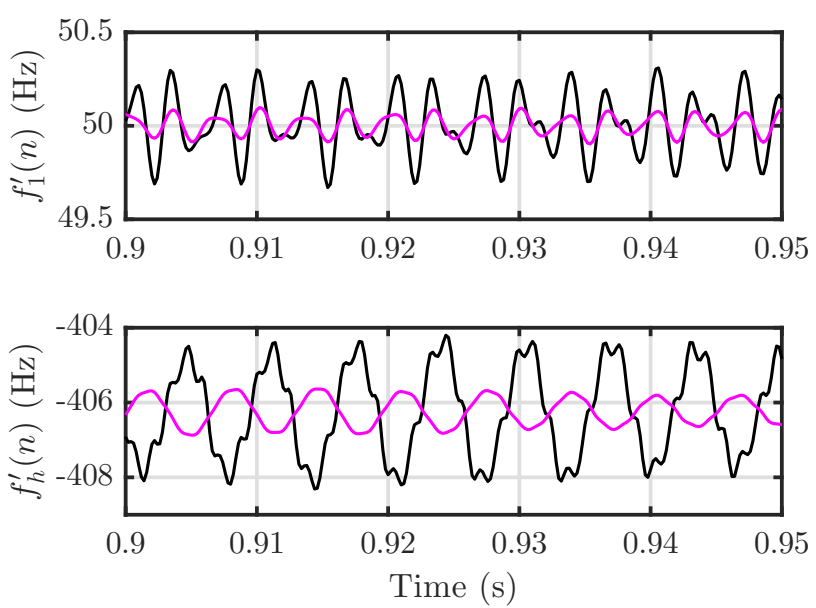

Fig. 14: Close-up of the CBF-FLL frequency estimations after the resonance has changed frequency for $\mathbf{Q}(z)$ (-) and $\mathbf{Q}^{2}(z)(-)$.

adaption rate that was invariant to the input signal magnitude and discrete CBF form. Two industrial applications, voltage synchronization during a grid fault and unknown frequency extraction from a power system signal, implemented on an FPGA, were used to verify the $p$ th-order discrete CBF-FLLs. The second-order discrete CBF-FLL offered a good compromise between performance and computational burden, motivating its use in high-performance converter applications such as voltage synchronization, reference current calculation, active filtering/damping, active damping and data-driven adaption.

\section{REFERENCES}

[1] P. Rodríguez, et. al., "A stationary reference frame grid synchronization system for three-phase grid-connected power converters under adverse grid conditions," IEEE Transactions on Power Electronics, vol. 27, no. 1, pp. 99-112, Jan. 2012.

[2] P. Rodriguez, et. al., "Multiresonant frequency-locked loop for grid synchronization of power converters under distorted grid conditions," IEEE Transactions on Industrial Electronics, vol. 58, no. 1, pp. 127-138, Jan. 2011.

[3] H. Yi, X. Wang, F. Blaabjerg, and F. Zhuo, "Impedance analysis of SOGI-FLL-based grid synchronization," IEEE Transactions on Power Electronics, vol. 32, no. 10, pp. 7409-7413, Oct. 2017.

[4] J. G. Hwang, P. W. Lehn, and M. Winkelnkemper, "A generalized class of stationary frame-current controllers for grid-connected AC-DC converters," IEEE Transactions on Power Delivery, vol. 25, no. 4, pp. 2742-2751, Oct. 2010.

[5] M. Mojiri, M. Karimi-Ghartemani, and A. Bakhshai, "Time-domain signal analysis using adaptive notch filter," IEEE Transactions on Signal Processing, vol. 55, no. 1, pp. 85-93, Jan. 2007.

[6] D. Yazdani, et. al., "A nonlinear adaptive synchronization technique for grid-connected distributed energy sources," IEEE Transactions on Power Electronics, vol. 23, no. 4, pp. 2181-2186, Jul. 2008.

[7] M. Karimi-Ghartemani and M. R. Iravani, "A method for synchronization of power electronic converters in polluted and variable-frequency environments," IEEE Transactions on Power Systems, vol. 19, no. 3, pp. 1263-1270, Aug. 2004.

[8] M. Karimi-Ghartemani and H. Karimi, "Processing of symmetrical components in time-domain," IEEE Transactions on Power Systems, vol. 22, no. 2, pp. 572-579, May 2007.

[9] K. W. Martin, "Complex signal processing is not complex," IEEE Transactions on Circuits and Systems I: Regular Papers, vol. 51, no. 9, pp. 1823-1836, Sept. 2004.

[10] L. Harnefors, "Modeling of three-phase dynamic systems using complex transfer functions and transfer matrices," IEEE Transactions on Industrial Electronics, vol. 54, no. 4, pp. 2239-2248, Aug. 2007.
[11] X. Q. Guo and W. Y. Wu, "Simple synchronisation technique for threephase grid-connected distributed generation systems," IET Renewable Power Generation, vol. 7, no. 1, pp. 55-62, Jan. 2013.

[12] X. Guo, W. Wu, and Z. Chen, "Multiple-complex coefficient-filter-based phase-locked loop and synchronization technique for three-phase gridinterfaced converters in distributed utility networks," IEEE Transactions on Industrial Electronics, vol. 58, no. 4, pp. 1194-1204, Apr. 2011.

[13] S. G. Jorge, C. A. Busada, and J. A. Solsona, "Frequency adaptive discrete filter for grid synchronization under distorted voltages," IEEE Transactions on Industrial Electronics, vol. 27, no. 8, pp. 3584-3594, Aug. 2012.

[14] C. A. Busada, et. al., "Current controller based on reduced order generalized integrators for distributed generation systems," IEEE Transactions on Industrial Electronics, vol. 59, no. 7, pp. 2898-2909, Jul. 2012.

[15] M. Cespedes and J. Sun, "Mitigation of inverter-grid harmonic resonance by narrow-band damping," IEEE Journal of Emerging and Selected Topics in Power Electronics, vol. 2, no. 4, pp. 1024-1031, Dec. 2014.

[16] J. He, et. al., "A flexible harmonic control approach through voltagecontrolled DG-grid interfacing converters," IEEE Transactions on Industrial Electronics, vol. 59, no. 1, pp. 444-455, Jan. 2012.

[17] W. Li, X. Ruan, C. Bao, D. Pan, and X. Wang, "Grid synchronization systems of three-phase grid-connected power converters: a complexvector-filter perspective," IEEE Transactions on Industrial Electronics, vol. 61, no. 4, pp. 1855-1870, Apr. 2014

[18] A. G. Yepes, et. al., "Effects of discretization methods on the performance of resonant controllers," IEEE Transactions on Power Electronics, vol. 25 , no. 7 , pp. 1692-1712, Jul. 2010.

[19] P. A. Regalia, "A complex adaptive notch filter," IEEE Signal Processing Letters, vol. 17, no. 11, pp. 937-940, Nov. 2010.

[20] E. F. Fuchs and M. A. S. Masoum, "Power Quality in Power Systems and Electrical Machines," Elsevier Academic Press, 2008.

[21] X. Wang, F. Blaabjerg, and M. Liserre, "An active damper to suppress multiple resonances with unknown frequencies," 2014 IEEE Applied Power Electronics Conference and Exposition (APEC), Fort Worth, TX, pp. 2184-2191, March 2014.

[22] R. Teodorescu, M. Liserre and P. Rodriguez, "Grid Converters for Photovoltaic and Wind Power Systems," John Wiley \& Sons, 2011.

[23] J. Matas, et. al., "An adaptive prefiltering method to improve the speed/accuracy tradeoff of voltage sequence detection methods under adverse grid conditions," IEEE Transactions on Industrial Electronics, vol. 61, no. 5, pp. 2139-2151, May 2014

[24] C. M. Rader, "Digital filter design techniques in the frequency domain," Proceedings of the IEEE, vol. 55, no. 2, pp. 149-171, Feb. 1967.

[25] A. V. Oppenheim and R. W. Schafer, "Discrete-Time Signal Processing, 3rd ed.," Pearson Prentice Hall, 2010.

[26] J. V. Zaen, "Efficient Schemes for Adaptive Frequency Tracking and their Relevance for EEG and ECG," Ph.D. dissertation, École Polytechnique Fédérale de Lausanne, Switzerland, 2012.

[27] W. H. Press, B. P. Flannery, S. A. Teukolsky, and W. T. Vetterling, "Numerical Recipes in FORTRAN: The Art of Scientific Computing, 2nd ed," Cambridge University Press, p. 710, 1992.

[28] L. B. Jackson, "A correction to impulse invariance," IEEE Signal Processing Letters, vol. 7, no. 10, pp. 273-275, Oct. 2000.

[29] A. G. Yepes, et. al., "High-performance digital resonant controllers implemented with two integrators," IEEE Transactions on Power Electronics, vol. 26, no. 2, pp. 563-576, Feb. 2011.

[30] P. Regalia, "Adaptive IIR Filtering in Signal Processing and Control," Marcel Dekker Inc., New York, 1995.

[31] S. H. Rice, "The expected value of the ratio of correlated random variables," Texas Tech University, USA, July 2015. [Online]. Available: http://www.faculty.biol.ttu.edu/rice/ratio-derive.pdf. Accessed Jan. 14,2018

[32] P. Stoica and R. L. Moses, "Spectral Analysis of Signals," Prentice Hall, New Jersey, 2005.

[33] D. Roiu, et. al., "New stationary frame control scheme for threephase PWM rectifiers under unbalanced voltage dips conditions," IEEE Transactions on Industry Applications, vol. 46, no. 1, pp. 268-277, 2010.

[34] P. Kadlec, R. Grbić, and B. Gabrys, "Review of adaptation mechanisms for data-driven soft sensors," Computers \& Chemical Engineering, vol. 35, no. 1, pp. 1-24, Jan. 2011. 


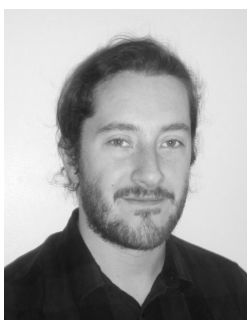

Emerson Guest received the Bachelor degree (2010) from the University of Newcastle, Newcastle, Australia, and the M.Sc. degree (2014) from the Technical University of Denmark, Kgs. Lyngby, Denmark, where he is currently working towards the Industrial Ph.D. degree, all in electrical engineering.

His research interests include modeling of grid-connected converters, active filtering and real-time optimization of power electronic systems.

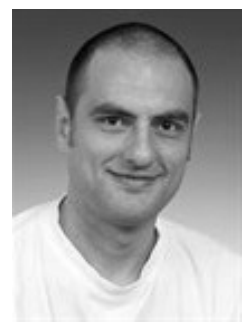

Nenad Mijatovic after obtaining his Dipl.Ing. education in Electrical Power Engineering at University of Belgrade, Serbia in 2007, was enrolled as a doctoral candidate at Technical University of Denmark. He received his Ph.D. degree from Technical University of Denmark for his work on technical feasibility of novel machines and drives for wind industry. Upon completion of his PhD, he continued work within the field of wind turbine direct-drive concepts as an Industrial PostDoc.

Dr. N. Mijatovic currently holds a position of Associate Professor at Technical University of Denmark where he is in charge of managing research projects and education related to the field of electrical machines and drives, motion control, and general applications of low frequency electromagnetism and large scale application of superconductivity with main focus on emerging eMobility and renewable energy generation. He is a member of IEEE since 2008 and senior member of IEEE since 2018 and his field of interest and research includes novel electrical machine/actuator designs, operation, control and diagnostic of electromagnetic assemblies, advance control of drives and grid connected power electronics, energy storage and eMobility. 